

\title{
A further study of reduced-order modeling techniques for nonlinear MEMS beams
}

\author{
Jérôme Juillard, Gabriel Vidal-Alvarez, Nuria Barniol
}

\section{To cite this version:}

Jérôme Juillard, Gabriel Vidal-Alvarez, Nuria Barniol. A further study of reduced-order modeling techniques for nonlinear MEMS beams. Design, Test, Integration and Packaging of MEMS/MOEMS (DTIP), 2015 Symposium on, Apr 2015, Monptellier, France. pp.1-6, 10.1109/DTIP.2015.7160983 . hal-01235620

\section{HAL Id: hal-01235620 \\ https://hal-centralesupelec.archives-ouvertes.fr/hal-01235620}

Submitted on 4 Dec 2015

HAL is a multi-disciplinary open access archive for the deposit and dissemination of scientific research documents, whether they are published or not. The documents may come from teaching and research institutions in France or abroad, or from public or private research centers.
L'archive ouverte pluridisciplinaire HAL, est destinée au dépôt et à la diffusion de documents scientifiques de niveau recherche, publiés ou non, émanant des établissements d'enseignement et de recherche français ou étrangers, des laboratoires publics ou privés. 


\section{A further study of reduced-order modeling techniques for nonlinear MEMS beams}

\author{
Jérôme Juillard \\ GEEPS (UMR 8507) \\ CentraleSupélec \\ Gif-sur-Yvette, FRANCE \\ Jerome.juillard@centralesupelec.fr
}

\author{
Gabriel Vidal-Álvarez, Nuria Barniol \\ Department of Electronic Engineering \\ Universitat Autònoma de Barcelona \\ Cerdanyola del Vallès, SPAIN
}

\begin{abstract}
This paper gives a further look at reduced-order modeling (ROM) techniques that can be applied to MEMS beams subject to nonlinear forces. It is focused on the popular method which consists in multiplying the equation governing the displacement of the beam by the displacement-dependent denominator of the nonlinear (electrostatic) force before modal projection is performed. Having already shown that in the case of 1-mode, 1-harmonic analysis, this method can lead to dramatically wrong results, we propose another choice of multiplicative coefficient, with much improved behavior. This method is illustrated, discussed and compared to other approaches in terms of simplicity, accuracy and range of validity.
\end{abstract}

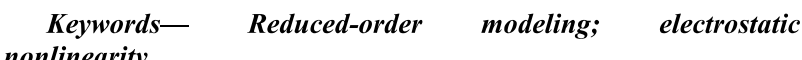

\section{INTRODUCTION}

Being able to accurately determine the nonlinear frequency response of a resonant M/NEMS device is of interest at several stages of a device's life, from the early design and modeling stage to the characterization or the calibration stage. For example, at the design stage, frequency responses are used as $a$ priori information to assess the characteristics (natural frequency, quality factor, influence of nonlinearity) of a structure. Experimental (nonlinear) frequency responses can be used to monitor the variations of these characteristics, for test, calibration or measurement. While the experimental determination of frequency responses is a problem unto itself [1-3], the issues raised by their theoretical determination should not be brushed aside. In [4], we compared three analysis techniques for tackling problems involving non-polynomial nonlinear forces. It turned out that, in the simple case of the single-sided electrostatic actuation of an otherwise linear beam, the popular method which consists in multiplying the equation governing the displacement of the beam by the displacementdependent denominator of the electrostatic force before modal projection is performed (MBP method, [5-6]), failed to capture:

- the down-shift of the resonance frequency caused by the DC bias, even at very small oscillation amplitudes.

- large amplitude effects such as the hysteretic characteristic of the frequency response.

On the other hand, these phenomena were qualitatively captured by straightforward Taylor series expansion of the nonlinear force (TS method, as in [7-8]), and quantitatively captured, even for very large displacements, by approximating the projection integrals with a function of similar asymptotic behavior (API method, as introduced in [9]). Note that several applications, such as characterization based on frequency response measurements, cannot rely on purely qualitative results.

In this paper, we consider, as in [4], the single-sided electrostatic actuation of an otherwise linear clamped-clamped beam, governed by:

$$
E I \frac{\partial^{4} w}{\partial x^{4}}+\mu \frac{\partial w}{\partial t}+\rho b h \frac{\partial^{2} w}{\partial t^{2}}=\frac{\varepsilon_{0} b}{2 G^{3}} \frac{V(t)^{2}}{(1-w)^{2}},
$$

where $G w(x, t)$ is the displacement, the beam has Young's modulus $E$, density $\rho$, length $L$, width $b$, electrostatic gap $G$, moment of inertia $I$, damping coefficient $\mu$, and $V(t)$ is the applied voltage. Note that the term describing the elongation of the beam is voluntarily omitted from (1). While, strictly speaking, it should be accounted for, it would only make our analysis more complex and our results more difficult to interpret. For the sake of clarity and brevity, we will only consider the simpler case described by (1).

Our objectives are (i) to shed a new light on why MBP, used as in [1], fails to capture nonlinear behavior, and (ii) to propose an alternative to MBP, actually another choice of multiplicative coefficient, with much improved behavior. In section II, we compare the frequency responses obtained, as in [4], from a single-mode, single-harmonic analysis of (1) through the TS and MBP approaches. The differences between the two expressions give us insight into why MBP can be highly inaccurate. In section III, we propose a modification of MBP that overcomes this issue. This method is illustrated, discussed and compared to other approaches in terms of simplicity, accuracy and range of validity. 
II. DETERMINATION OF NONLINEAR FREQUENCY RESPONSES THROUGH TS AND MBP

Letting $\xi=x / L$ and $\tau=\omega_{0} t$, where

$$
\omega_{0}^{2}=\frac{\lambda^{4} E I}{\rho b h L^{4}}, \lambda \approx 4.73,
$$

supposing $V(\tau)=V_{b}\left(1+v_{0} \cos (\omega \tau)\right)$, where $v_{0}<<1$, and $w(\xi, \tau) \approx a(\tau) w_{C C}(\xi)$ with $w_{C C}(\xi)$ the first clampedclamped eigenmode, equation (1) can be rewritten as:

$$
\left(a+\frac{\dot{a}}{Q}+\ddot{a}\right) w_{C C}=\eta \frac{1+2 v_{0} \cos (\omega \tau)}{\left(1-a w_{C C}\right)^{2}}, \eta=\frac{\varepsilon_{0} V_{b}^{2}}{2 \rho h G^{3} \omega_{0}^{2}}
$$

where the dot denotes differentiation with respect to $\tau, \eta$ is an electromechanical coupling coefficient and $Q$ is the quality factor.

\section{A. TS method}

The right-hand side of (3) is expanded assuming $a w_{C C}<<1$. For a third order expansion, this yields:

$$
\begin{aligned}
& \left(a+\frac{\dot{a}}{Q}+\ddot{a}\right) w_{C C}= \\
& \eta\left(1+2 v_{0} \cos (\omega \tau)\right)\left(1+2 a w_{C C}+3\left(a w_{C C}\right)^{2}+4\left(a w_{C C}\right)^{3}\right)
\end{aligned}
$$

Projection on $w_{C C}$ results in the transient 1 degree-of-freedom model:

$$
\begin{aligned}
& \left(a+\frac{\dot{a}}{Q}+\ddot{a}\right) I_{1}= \\
& \eta\left(1+2 v_{0} \cos (\omega \tau)\right)\left(I_{0}+2 a I_{1}+3 a^{2} I_{2}+4 a^{3} I_{3}\right)
\end{aligned}
$$

$$
\text { where } \quad I_{k}=\int_{0}^{1}\left(w_{C C}\right)^{k+1} d \xi \quad\left(I_{0} \approx 0.523, \quad I_{1} \approx 0.397\right. \text {, }
$$

$I_{2} \approx 0.332$ and $I_{3} \approx 0.291$ ).

The steady-state model is obtained by assuming that $a(\tau)=A \sin (\omega \tau+\varphi)$ and projecting (5) on $\sin (\omega \tau+\varphi)$ and $\cos (\omega \tau+\varphi)$ (i.e. by balancing over one period [10]). This yields the following equations:

$$
\left\{\begin{array}{c}
A\left(\left(1-\omega^{2}\right)-\eta\left(2+3 \frac{I_{3}}{I_{1}} A^{2}\right)\right)=-2 \eta v_{0} \sin \varphi \frac{I_{0}}{I_{1}}\left(1+\frac{9}{4} \frac{I_{2}}{I_{0}} A^{2}\right) \\
A \frac{\omega}{Q}=2 \eta v_{0} \cos \varphi \frac{I_{0}}{I_{1}}\left(1+\frac{3}{4} \frac{I_{2}}{I_{0}} A^{2}\right)
\end{array}\right.
$$

One may then express $v_{0}$ as a function of $A$ and $\omega$ by eliminating $\sin \varphi$ and $\cos \varphi$ from (6) and plot contour lines of $v_{0}$ vs. $A$ and $\omega$ using numerical approximation methods (e.g. the contour function in Matlab). This yields the frequency response of the system for various values of $v_{0}$.

\section{B. MBP method}

Equation (3) is multiplied by $\left(1-a w_{C C}\right)^{2}$, yielding:

$$
\left(a+\frac{\dot{a}}{Q}+\ddot{a}\right) w_{C C}\left(1-a w_{C C}\right)^{2}=\eta\left(1+2 v_{0} \cos (\omega \tau)\right)
$$

and projected on $w_{C C}$. This results in the 1-dof model:

$$
\left(a+\frac{\dot{a}}{Q}+\ddot{a}\right)\left(I_{1}-2 a I_{2}+a^{2} I_{3}\right)=\eta\left(1+2 v_{0} \cos (\omega \tau)\right) I_{0} .
$$

The method of harmonic balance then yields:

$$
\left\{\begin{array}{c}
A\left(1-\omega^{2}\right)\left(1+\frac{3}{4} \frac{I_{3}}{I_{1}} A^{2}\right)=-2 \eta v_{0} \sin \varphi \frac{I_{0}}{I_{1}} \\
A \frac{\omega}{Q}\left(1+\frac{1}{4} \frac{I_{3}}{I_{1}} A^{2}\right)=2 \eta v_{0} \cos \varphi \frac{I_{0}}{I_{1}}
\end{array},\right.
$$

from which the frequency response can be plotted, as in II-A.

C. Qualitative and quantitative comparison of TS and MBP

Despite the general similarity of (6) and (9), there is a very large qualitative difference between the two expressions. This can be emphasized by determining how the resonance frequency changes with amplitude. To this end, we let $\varphi=0$ in (9), which yields:

$$
\omega=1 .
$$

The same operation in (6) yields:

$$
\omega=\sqrt{1-\eta\left(2+3 \frac{I_{3}}{I_{1}} A^{2}\right)}
$$

In other words, the MBP method predicts a constant resonant frequency, independent of the excitation amplitude $v_{0}$ or of the DC bias $\eta$, as opposed to the TS method. This was illustrated in [4].

This behavior of MBP is in fact not so surprising: it is known that, if $a(\tau)$ is represented with one harmonic only, the method of harmonic balance will not take even nonlinearities into account [11]. Thus, the DC bias term appearing on the right-hand side of (8), being independent of $a(\tau)$, has no 
influence on the frequency response predicted with MBP, as (10) shows. On the contrary, the electrostatic softening phenomenon is predicted by the TS approach thanks to the odd nonlinear terms on the right-hand side of (5). Moreover, we know that, for very small oscillation amplitudes, the resonance frequency is accurately predicted by (11). These considerations must be taken into account in order to construct an "improved" MBP method.

\section{Construction of an improved MBP method}

Several avenues can be pursued in order to improve the results obtained with MBP. Sticking to harmonic balance, an obvious development would be to increase the number of harmonics used for representing $a(\tau)$. However, this leads to very complex expressions from which it becomes difficult to extract a frequency response. Moreover, the fact that the motion of an electrostatic oscillating beam can be accurately described with only one mode and one harmonic is confirmed experimentally [12]. So increasing the number of modes or of harmonics goes against empirical fact.

One may also look for more adequate basis functions for the representation of $w$ and/or for the projection of the equation resulting from MBP (e.g. (7)): this is somehow justified, since, through MBP, the differential operator appearing in (1) becomes highly nonlinear and the beam eigenmodes are not as relevant as in the linear case. Although this approach might be worth considering for improving the results of MBP in the static case, straightforward calculations show that it would have no qualitative consequence on the predicted frequency responses. Yet another possibility is to look for a more complex, but hopefully more appropriate multiplicative coefficient than $(1-w)^{2}$. The new multiplicative coefficient should have the same benefits as the old one (solving for the oscillation amplitude should remain a polynomial problem), and it should also capture softening at least as well as Taylor series expansion. In other words, one may improve the results of MBP by choosing as a multiplicative coefficient:

$$
P(w)(1-w)^{2}
$$

where $P(w)$ verifies:

$$
P(w)=1+2 w+\text { h.o.t. }
$$

so that the two lowest-degree terms of $P(w)$ match those of the Taylor series expansion of $(1-w)^{-2}$. Although there are infinitely many polynomials meeting this criterion, we find that

$$
P(w)=1+2 w+w^{2}=(1+w)^{2},
$$

is both convenient and accurate. This choice of $P(w)$ means that the improved MBP consists in multiplying both sides of (1) by $\left(1-w^{2}\right)^{2}$. Assuming a single mode representation of displacement $w$, this yields:

$$
\begin{aligned}
& \left(a+\frac{\dot{a}}{Q}+\ddot{a}\right) w_{C C}\left(1-\left(a w_{C C}\right)^{2}\right)^{2} . \\
& =\eta\left(1+2 v_{0} \cos (\omega \tau)\right)\left(1+a w_{C C}\right)^{2}
\end{aligned}
$$

which, when projected on $w_{C C}$, results in the 1-dof model:

$$
\begin{aligned}
& \left(a+\frac{\dot{a}}{Q}+\ddot{a}\right)\left(I_{1}-2 a^{2} I_{3}+a^{4} I_{5}\right) \\
& =\eta\left(1+2 v_{0} \cos (\omega \tau)\right)\left(I_{0}+2 a I_{1}+a^{2} I_{2}\right)
\end{aligned}
$$

Thus, the first two terms on the right-hand side do correspond to those obtained with the TS approach (5): this guarantees an accurate static response and resonance frequency for small displacements. Furthermore, only odd nonlinearities appear on the left-hand side of (16), which ensures that 1-harmonic harmonic balancing will take into account the effect of nonlinearity. Finally, it is worth noting that the proposed multiplicative coefficient is the one that naturally arises in double-sided configurations, when the resonator is placed between two equidistant electrodes.

The frequency response is obtained by using the method of harmonic balance on (16). This yields:

$$
\left\{\begin{array}{c}
A\left[\left(1-\omega^{2}\right)\left(1-\frac{3}{2} \frac{I_{3}}{I_{1}} A^{2}+\frac{5}{8} \frac{I_{5}}{I_{1}} A^{4}\right)-2 \eta\right] \\
=-2 \eta v_{0} \sin \varphi \frac{I_{0}}{I_{1}}\left(1+\frac{3}{4} \frac{I_{2}}{I_{0}} A^{2}\right) \\
A \frac{\omega}{Q}\left(1-\frac{1}{2} \frac{I_{3}}{I_{1}} A^{2}+\frac{1}{8} \frac{I_{5}}{I_{1}} A^{4}\right) \\
=2 \eta v_{0} \cos \varphi \frac{I_{0}}{I_{1}}\left(1+\frac{1}{4} \frac{I_{2}}{I_{0}} A^{2}\right)
\end{array} .\right.
$$

from which the frequency response can be plotted, as in II-A.

\section{E. API method}

Equation (3) is projected on $w_{C C}$ :

$$
\left(a+\frac{\dot{a}}{Q}+\ddot{a}\right) I_{1}=\eta\left(1+2 v_{0} \cos (\omega \tau)\right) \int_{0}^{1} \frac{w_{C C}}{\left(1-a w_{C C}\right)^{2}} d \xi
$$

The right hand-side is approximated, following [9], as: 


$$
\int_{0}^{1} \frac{w_{C C}}{\left(1-a w_{C C}\right)^{2}} d \xi \approx I_{0} \frac{1+\kappa a}{(1-a)^{3 / 2}}
$$

The choice of $\kappa$ depends on the criterion used for fitting both sides of (18). In [4], a coefficient of $2.15 \times 10^{-2}$ was used, determined numerically to yield the smallest relative error for $0 \leq a \leq 0.99$. Alternatively, we may choose $\kappa$ so that the first two terms of the TS expansion of the electrostatic force are matched. This yields:

$$
\kappa=2 \frac{I_{1}}{I_{0}}-\frac{3}{2} \approx 1.56 \times 10^{-2}
$$

The method of harmonic balance yields:

$$
\left\{\begin{array}{c}
A\left(1-\omega^{2}\right)-\eta \frac{I_{0}}{I_{1}} f(A)=-2 \eta v_{0} \sin \varphi \frac{I_{0}}{I_{1}} g(A) \\
A \frac{\omega}{Q}=2 \eta v_{0} \cos \varphi \frac{I_{0}}{I_{1}} g(A)
\end{array}\right.
$$

where

$$
\begin{gathered}
f(A)=\frac{4}{\pi A}\left[\begin{array}{c}
(1+\kappa) \frac{\sqrt{1+A}}{1-A^{2}}(\mathrm{E}-(1-A) \mathrm{K}) \\
-\frac{\kappa}{\sqrt{1+A}}(\mathrm{~K}-(1+A) \mathrm{E})
\end{array}\right], \\
g(A)=\frac{8}{\pi A^{2}}\left[\begin{array}{c}
\frac{(1+\kappa)}{\sqrt{1+A}}(\mathrm{~K}-(1+A) \mathrm{E}) \\
-\frac{\kappa}{3} \sqrt{1+A}(\mathrm{E}-(1-A) \mathrm{K})
\end{array}\right],
\end{gathered}
$$

and $\mathrm{K}$ (resp. E) stands for the complete elliptic integral of the first (resp. second) kind, with argument $2 A /(1+A)$. Even though (22-23) are not polynomials in $A$, it is not costly to evaluate them. The frequency response is then easily obtained by drawing the contour lines of $v_{0}$.

\section{RESULTS}

\section{A. Comparison of $M B P$ and improved MBP methods}

First of all, let us illustrate the qualitative improvement introduced with the proposed approach over the MBP method. We plot in Fig. 1 the frequency responses computed with (9) and (17) for $Q=500, \eta=0.01$ and different values of the
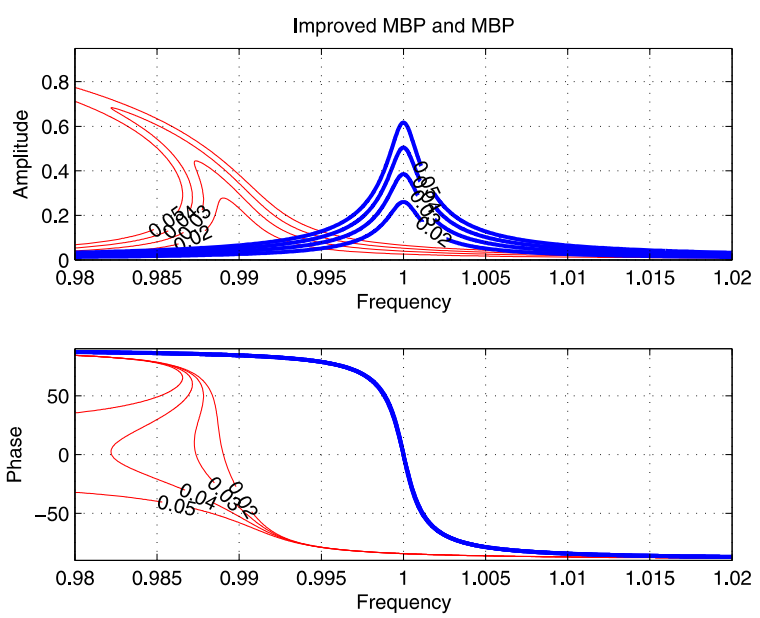

Fig. 1. Comparison of frequency responses obtained with the MBP method (thick lines) and the improved MBP method (thin lines).

actuation voltage $\left(0.02 \leq v_{0} \leq 0.05\right)$. These parameter values remain unchanged throughout section III. This shows clearly that the frequency response determined with the improved MBP method does capture electrostatic softening, as opposed to the classical MBP method.

\section{B. Comparison of improved MBP, API and TS methods}

A first measure of the accuracy of the improved MBP method consists in comparing it with the TS approach. The frequency responses obtained with improved MBP (17), and with a $3^{\text {rd }}$ order TS expansions (6) are represented in Fig. 2. As expected, for small and moderate amplitudes, the results obtained with the improved MBP and TS methods are consistent. On the other hand, notable discrepancies start to appear at large oscillation amplitudes (about one third of the gap). Although only the results obtained with $3^{\text {rd }}$-order Taylor expansion are reported here, increasing the order of the series does not seem to reduce discrepancy. Similar discrepancies can be observed at large oscillation amplitudes between the improved MBP and API methods (Fig. 3), with $\kappa=1.56 \times 10^{-2}$.

In our simulations, we observe that the nonlinear behavior is always more marked in frequency responses predicted with API than in those predicted with improved MBP. The same goes for improved MBP compared to TS.

To estimate the accuracy of each method, one may compare the actuation voltage calculated at given oscillation amplitudes and oscillation frequencies through (i) improved MBP (17), (ii) API (23) and (iii) direct use of harmonic balance and modal projection on (3) through double numerical quadrature (although the procedure is quite costly). The latter approach is used as a reference method and the relative error on the actuation voltages found with API and improved MBP are plotted in Fig. 4 and 5. The error obtained with API stays below $3 \%$ on the whole studied range, while that of improved MBP is much higher, especially at large oscillation amplitudes, where it may exceed $50 \%$. 



Fig. 2. Comparison of frequency responses obtained with the TS method (thick lines)and the improved MBP method (thin lines).
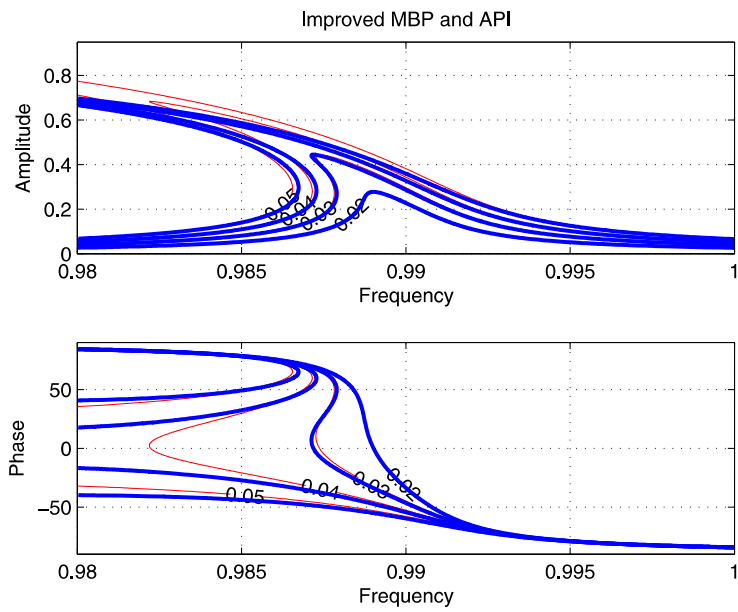

Fig. 3. Comparison of frequency responses obtained with the API method (thick lines) and the improved MBP method (thin lines).

\section{DISCUSSION}

Several conclusions may be drawn from our study. First of all, it is possible to choose a multiplicative coefficient in the MBP method which yields qualitatively good results if used in conjunction with harmonic balance. The choice of this coefficient is not unique. A physically-meaningful one is the one that naturally arises in evenly-spaced double-sided configurations.

If this choice is made, the resulting steady-state model has a similar complexity to the one obtained through a $3^{\text {rd }}$ order TS expansion, but is more accurate. On the other hand, it is much less accurate, at large oscillation amplitudes, than the admittedly more complex steady-state model obtained with API. Consequently, one should prefer API to other methods when looking for quantitatively sound results, for example when trying to characterize a structure from its nonlinear frequency response.

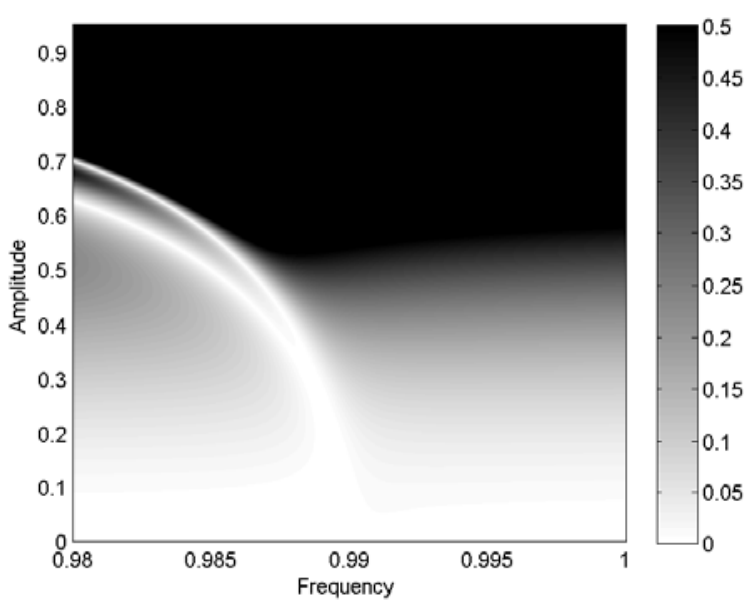

Fig. 4. Relative error on v0 between improved MBP and reference method.

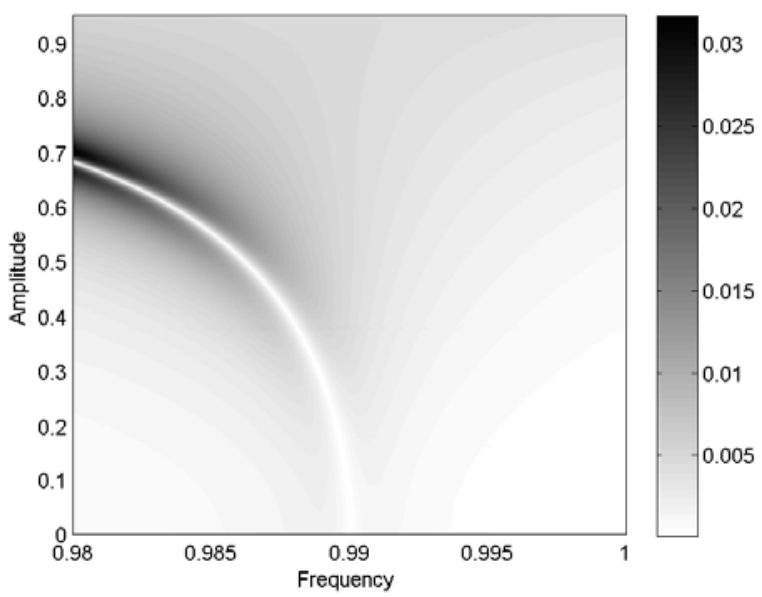

Fig. 5. Relative error on v0 between API and reference method.

Several questions remain open, in particular concerning the extension of the MBP to more complex cases, e.g. when other nonlinearities are present (stress-stiffening, squeezed-fim damping) or asymmetrical double-sided configurations. Another point worth investigating is how to take into account the constant (DC) component of the displacement, that was neglected throughout this work, and which may in fact play an important role in asymmetrical configurations, especially at large oscillation amplitudes, as theory and simulations show [13].

\section{ACKNOWLEDGMENT}

This work has been partially funded by the Spanish Government and the European Union FEDER program under project TEC2012-32677 (NEMS-in-CMOS).

\section{REFERENCES}

[1] A.T.H. Lin et al., "Enhanced transduction methods for electrostatically driven MEMS resonators", Solid-State Sensors, Actuators and Microsystems Conference, pp. 561-564, 2009 
[2] J.E.Y. Lee, A.A. Seshia, "Direct parameter extraction in feedthroughembedded capacitive MEMS resonators", Journal of Micromechanics and Microengineering, vol. 167, pp. 237-244, 2011

[3] A. Brenes et al., "Characterization of MEMS resonators via feedthrough de-embedding of pulsed-mode response", Proceedings of Eurosensors, 2014, accepted.

[4] J. Juillard, "A comparative study of reduced-order modeling techniques for nonlinear MEMS beams", Proceedings of DTIP, pp. 261-265, 2014.

[5] M. I. Younis et al., "A reduced-order model for electrically actuated microbeam-based MEMS", Journal of Microelectromechanical Systems, vol. 12 , pp. $672-680,2003$

[6] A. H. Nayfeh et al., "Reduced-order models for MEMS applications", Nonlinear Dynamics, vol. 41, pp. 211-236, 2005

[7] J. F. Rhoads et al., "Generalized parametric resonance in electrostatically actuated microelectromechanical oscillators", Journal of Sound and Vibration, vol. 296, pp. 797-829, 2006
[8] A. A. Trusov et al., "Capacitive detection in resonant MEMS with arbitrary amplitude of motion", Journal of Micromechanics and Microengineering, vol. 17, pp. 1583-1592, 2007

[9] J. Juillard et al., "Modeling of micromachined beams subject to nonlinear restoring or damping forces", Journal of Microelectromechanical Systems, vol. 20, pp. 165-177, 2011

[10] A. Gelb, W. Van der Velde, "Multiple-input describing functions and nonlinear system design", McGraw-Hill, New-York, 1968

[11] R. Porwal, N.S. Vyas, "Damped quadratic and mixed-parity oscillator response using Krylov-Bogoliubov method and energy balance", Journal of Sound and Vibration, vol. 309, pp. 877-866

[12] A. Brenes et al., "Parameter estimation from nonlinear frequency response of MEMS resonators", International Symposium on Circuits and Systems, 2015, submitted

[13] J. Juillard, "Analysis of resonant pull-in of micro-electromechanical oscillators", Journal of Sound and Vibration, 2015, submitted 